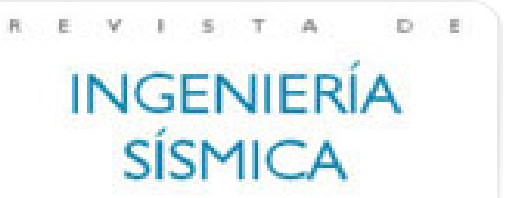

Revista de Ingeniería Sísmica

ISSN: 0185-092X

javiles@tlaloc.imta.mx

Sociedad Mexicana de Ingeniería Sísmica

México

Álvarez, José de Jesús; Jara, Manuel; Hernández, Hugo; Aparicio, Ángel Carlos Evaluación de la respuesta de grandes puentes en arco ante movimientos sísmicos fuertes

Revista de Ingeniería Sísmica, núm. 75, 2006, pp. 23-46

Sociedad Mexicana de Ingeniería Sísmica

Distrito Federal, México

Disponible en: http://www.redalyc.org/articulo.oa?id=61807502

- Cómo citar el artículo

- Número completo

- Más información del artículo

- Página de la revista en redalyc.org

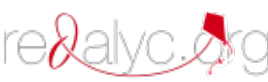

Sistema de Información Científica

Red de Revistas Científicas de América Latina, el Caribe, España y Portugal Proyecto académico sin fines de lucro, desarrollado bajo la iniciativa de acceso abierto 


\title{
EVALUACIÓN DE LA RESPUESTA DE GRANDES PUENTES EN ARCO ANTE MOVIMIENTOS SÍSMICOS FUERTES
}

\author{
José de Jesús Álvarez, Manuel Jara, Hugo Hernández ${ }^{(1)}$ \\ Ángel Carlos Aparicio ${ }^{(2)}$
}

\begin{abstract}
RESUMEN
Se estudia el comportamiento inelástico de puentes en arco de concreto sometidos a movimientos sísmicos fuertes, mediante una serie de análisis dinámicos de un puente de gran claro usando un modelo tridimensional. El modelo corresponde a un puente con tablero superior de $600 \mathrm{~m}$ de longitud total y un claro principal de $400 \mathrm{~m}$; se tiene en cuenta el comportamiento no lineal de los arranques del arco y de los extremos de las pilas, zonas de potenciales articulaciones plásticas. Para evaluar la respuesta se usaron 10 conjuntos de registros escalados con tres componentes cada uno. Los resultados muestran que las mayores respuestas del modelo fueron producidas por los sismos con mayores velocidades máximas del terreno; las demandas de ductilidad excedieron la capacidad rotacional en los arranques del arco para movimientos transversales. Se obtuvo que la compresión en los arranques del arco varía significativamente durante la ocurrencia de los movimientos, por lo que debe considerarse la interacción no lineal entre la compresión y el momento flexionante. Al estudiar la variabilidad espacial se encontró que las demandas rotacionales en los arranques del arco aumentaron para flexión en el plano, debido a una mayor respuesta vertical del modelo.
\end{abstract}

\begin{abstract}
The inelastic behavior of concrete arch bridges subjected to strong ground motions is investigated by dynamic analyses of a long-span arch bridge using a three-dimensional analytical model. The model considered is an upper-deck concrete arch bridge with a main span of $400 \mathrm{~m}$ and a total length of $600 \mathrm{~m}$. The non-linear behavior is considered by means of plastic hinges located at the arch springings and at the ends of the piers. Ten three-component scaled seismic ground motion records were used as inputs for the simulation analysis. According to analyses results, higher responses were obtained for records with maximum ground velocities; rotational ductility demands were greater than rotational capacities of plastic hinges located at both springings of the arch for transverse seismic excitation. Axial force on the arch springings experimented significant variations during the seismic excitation; as a consequence, this effect must be considered for the evaluation of plastic hinge properties. Potential problems associated with asynchronous ground motions at the springings of the arch were evaluated; results of the space variability analyses shown a rotational demand increment at both arch springings because of the higher vertical response of the model.
\end{abstract}

Artículo recibido el 25 de octubre de 2005 y aprobado para su publicación el 7 de abril de 2006. Se aceptarán comentarios y/o discusiones hasta cinco meses después de su publicación.

(1) Facultad de Ingeniería Civil, Universidad Michoacana de San Nicolás de Hidalgo. Edificio de Posgrado de Ingeniería Civil, Ciudad Universitaria, 58030, Morelia, Mich.asereno@umich.mx, mjara@umich.mx, hugohbarrios@yahoo.com.mx

(2) Departamento de Ingeniería de la Construcción, Universidad Politécnica de Cataluña. angel.carlos.aparicio@upc.edu 


\section{INTRODUCCIÓN}

Gracias al desarrollo de nuevos métodos constructivos y al avance en la tecnología de los materiales, los puentes en arco resultan competitivos en un intervalo de claros que oscila entre los 200 y los 600 metros (Muller, 2001). Los actuales récords mundiales son el Puente Wanxian, con un claro principal de $420 \mathrm{~m}$, y el Puente Lupu, con un claro principal de $550 \mathrm{~m}$, para puentes de concreto y de acero, respectivamente (Virola, 2005), aunque se han llegado a proyectar arcos de hasta $600 \mathrm{~m}$ de claro (Spielmann, 2001).

Los puentes en arco son estructuras que exhiben un comportamiento complejo durante la ocurrencia de temblores fuertes. El arco es un elemento estructural que se ve sometido a una elevada fuerza de compresión generada por la carga permanente, lo que lo hace un elemento con ductilidad limitada; además, dicha fuerza puede variar de manera importante durante la ocurrencia de un movimiento sísmico. Por lo anterior, resulta de interés conocer las demandas de ductilidad a que puede verse sometido este elemento principal y la posible redistribución de las demandas en otros elementos del puente.

Aun cuando los puentes en arco no han sufrido daño severo en el pasado reciente, existen algunos que han quedado sísmicamente deficientes ante los códigos actuales de diseño y que han sido motivo de estudio, como el Puente del Bixby Creek en la costa de California (McCallen et al, 1999) y los Puentes Krk I y II, construidos en 1980 en Croacia (Simunic et al, 2002); vale la pena recordar que el Puente Krk I fue récord mundial de los puentes en arco de concreto con sus 390 metros de claro principal.

En los últimos 20 años se han llevado a cabo algunas investigaciones tendientes a esclarecer la respuesta sísmica de puentes en arco. Los puentes de acero han sido estudiados recientemente por Torkamani y Lee (2002), Nazmy (2003) y Usami et al, (2004). Respecto a los de concreto reforzado son de destacar los trabajos realizados por McCallen et al, (1999), Kawashima y Misoguti (2000), y Sakai y Kawashima (2002). Un estado del arte sobre el comportamiento de puentes en arco ante acción sísmica se presenta en Álvarez y Aparicio (2003). No obstante lo anterior, los estudios relativos al tema son escasos; en particular, el comportamiento no lineal de grandes puentes en arco de concreto no ha sido estudiado. Para diseñar puentes en arco con una seguridad razonable o desarrollar lineamientos que permitan estimar los daños, es esencial que el diseñador cuente con información relativa a su comportamiento tanto ante carga gravitacional como ante acciones sísmicas.

Con la finalidad de contribuir al estudio de estas complejas estructuras, en esta investigación, se estudia el comportamiento inelástico de un puente en arco de concreto reforzado, sometido a movimientos sísmicos fuertes. Se trata de un puente en arco con tablero superior, modelado en 3D usando el programa de elementos finitos SAP2000 (CSI, 1997). Los acelerogramas usados son registros escalados de los sismos de Campano-Lucano (Italia, 1980), Michoacán (México, 1985), Northridge (EUA, 1994), Kobe (Japón, 1995), y Chi-Chi (Taiwán, 1999). Se evalúan las características dinámicas del puente en sus tres direcciones principales y se investigan las respuestas temporales de los parámetros principales, como son los desplazamientos longitudinales, transversales y verticales, la fluctuación de la fuerza axial y las demandas de 
ductilidad en determinados sitios de la subestructura. Los resultados muestran que las mayores respuestas del modelo fueron producidas por los movimientos con mayor velocidad máxima del terreno; en particular, los arranques del arco se vieron sometidos a mayores demandas de ductilidad para movimientos en dirección transversal debido a la plastificación de estas zonas. Finalmente, se presenta una evaluación del comportamiento con base en normas internacionales de diseño sísmico de puentes, atendiendo a la capacidad rotacional de las articulaciones plásticas de la estructura.

\section{MODELO ANALÍTICO}

En la figura 1 se muestra una vista en elevación del puente, dimensionado ex profeso para esta investigación (Álvarez, 2004). Se trata de un puente en arco de concreto con tablero superior, con una longitud total de $600 \mathrm{~m}$, subdividida en 10 claros parciales de $40 \mathrm{~m}$ sobre el arco, cuatro claros de $35 \mathrm{~m}$ (dos a cada lado del arco) y dos claros de $30 \mathrm{~m}$ (uno en cada extremo). La superestructura está formada por un tablero continuo que se apoya en dos estribos y 15 pilas. El arco, cuya directriz se aproxima bastante a la curva antifunicular de las cargas permanentes, salva un claro de $400 \mathrm{~m}$ y tiene una flecha de $80 \mathrm{~m}$, para una relación flecha a claro de 1/5.

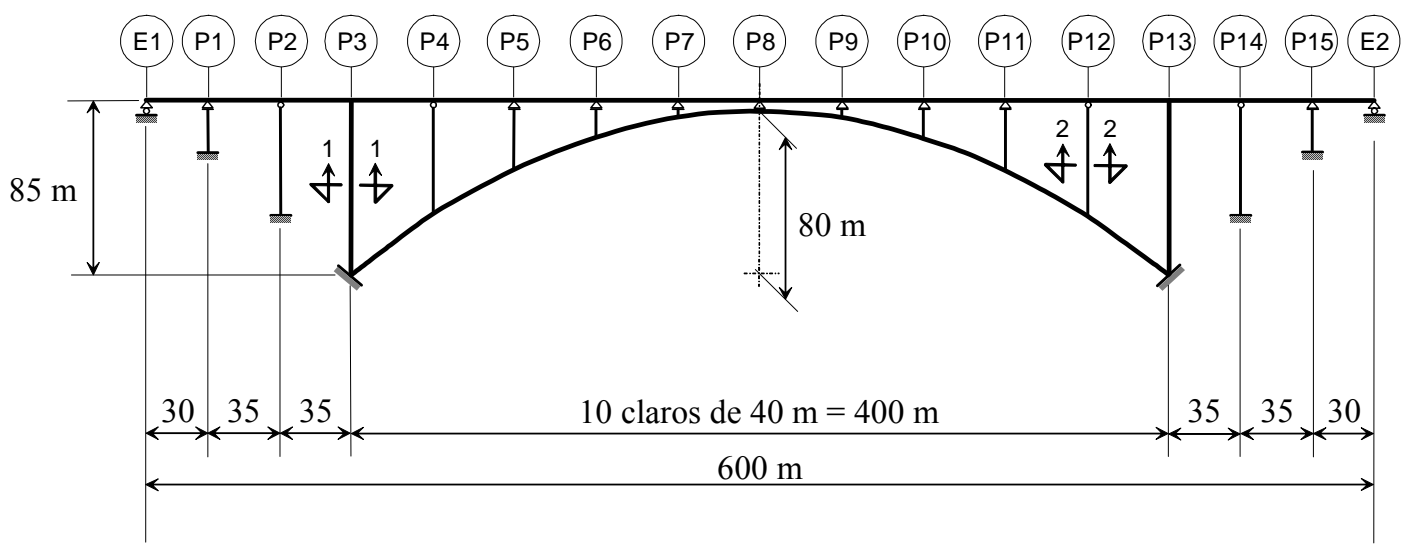

Figura 1. Vista en elevación del modelo

En la figura 2 se presentan las secciones transversales de los elementos principales. El tablero es un cajón monocelular con doble voladizo de $20 \mathrm{~m}$ de anchura total (carretera de cuatro carriles) y $2.5 \mathrm{~m}$ de peralte. El arco es de sección rectangular en cajón bicelular de $15 \mathrm{~m}$ de anchura y peralte variable desde $7 \mathrm{~m}$ en los arranques hasta $5.5 \mathrm{~m}$ en las secciones localizadas a $60 \mathrm{~m}$ de los arranques medidos en dirección longitudinal; entre estas secciones el peralte es constante de $5.5 \mathrm{~m}$. Las pilas P3 y P13 tienen una altura de $85 \mathrm{~m}$ y constan de una sección cajón monocelular de 6 por $10 \mathrm{~m}$. El resto de las pilas, de altura variable, consta de una sección cajón bicelular de 3 por $10 \mathrm{~m}$.

Con relación a las condiciones de apoyo, las pilas P1 a P3, P13 a P15, y los arranques del arco, se consideraron empotrados en la cimentación, debido a que este tipo de puentes generalmente se construye sobre suelo duro o roca y es de esperarse que la interacción suelo- 
estructura tenga poca influencia en la respuesta estructural. En cuanto a la vinculación pilastablero, las pilas P3 y P13, que en lo sucesivo serán identificadas como pilas principales, son continuas con la superestructura. Las pilas adyacentes a las principales (P2, P4, P12 y P14) se consideraron articuladas en su conexión con la superestructura. En el resto de las pilas (P1, P5 a P11, y P15), de menor longitud, y en ambos estribos, se consideró que el tablero descansa sobre apoyos que permiten el deslizamiento horizontal del tablero (PTFE multidireccionales, figura 2 a). Asimismo, se supuso que todas las pilas con apoyos deslizantes y ambos estribos estarían dotados de llaves de cortante capaces de tomar los desplazamientos transversales de la superestructura. Por otro lado, todas las pilas apoyadas sobre el arco se consideraron continuas con éste. Como consecuencia, despreciando la fricción en los apoyos deslizantes que están dotados de discos de PTFE (teflón), las pilas P2, P3, P4, P12, P13 y P14 en dirección longitudinal, y el arco, todas las pilas y ambos estribos en direcciones transversal y vertical resistirían las fuerzas de inercia de la superestructura.

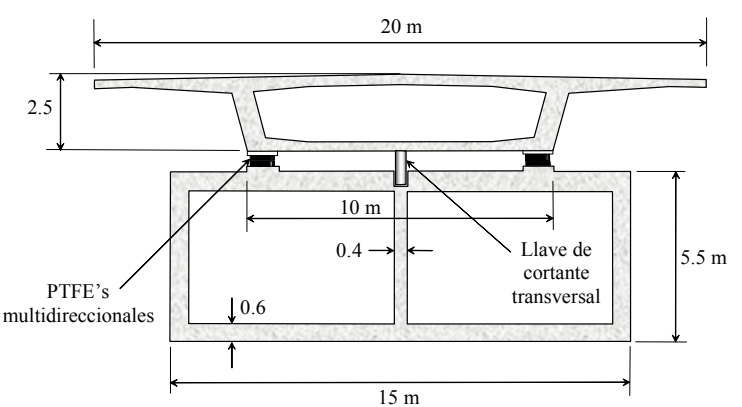

a). Arco y tablero en el centro del claro

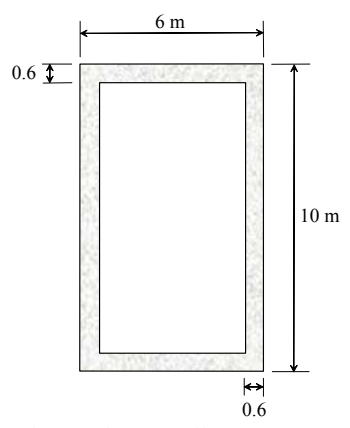

b). Sección 1-1. Pilas P3 y P13

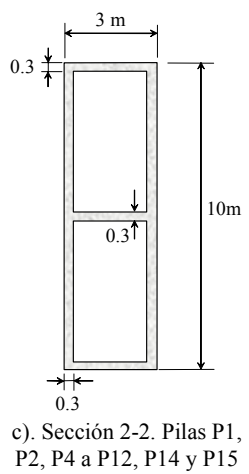

Figura 2. Secciones transversales del arco, tablero y pilas

El modelo del puente se elaboró empleando la versión no lineal del programa de elementos finitos SAP2000. En general, se emplearon elementos barra tridimensional (tipo frame en SAP2000)) para modelar los diferentes elementos estructurales. En la figura 3 se muestra el esquema bidimensional del puente en arco, el cual cuenta con 966 grados de libertad.

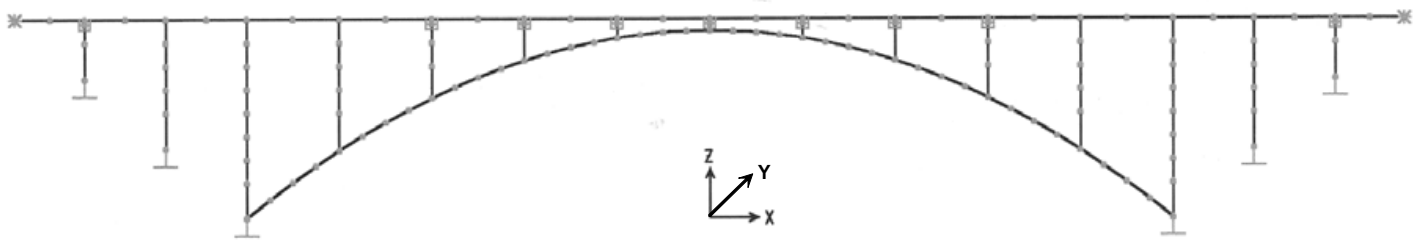

Figura 3. Modelo analítico

Las pilas se modelaron mediante elementos prismáticos tipo frame. En las pilas de más de $20 \mathrm{~m}$ de altura se consideraron nudos intermedios con separaciones entre 10 y $15 \mathrm{~m}$, dado que los modos superiores podrían contribuir significativamente a su respuesta dinámica. Para tomar en cuenta el agrietamiento por flexión, que es el primero en aparecer, los momentos de inercia 
efectivos de las secciones transversales se supusieron iguales al $70 \%$ de los correspondientes a las secciones brutas (Abeysinghe et al, 2002). El área, la constante de torsión y las áreas de cortante se calcularon con base en la sección bruta. Además, se emplearon dos conjuntos de elementos finitos en los extremos superiores de las pilas (cabezales) equipadas con apoyos deslizantes (figura 4): 1) elementos tipo nllink, que permitiesen modelar el deslizamiento del tablero sobre las pilas en dirección longitudinal, y 2) elementos rígidos tipo frame, para conectar las placas de apoyo con el centro de gravedad de la superestructura. Mediante la opción member releases se liberó el grado de libertad de giro de los cabezales de las pilas P2, P4, P12 y P14 (figura 1), articuladas con el tablero.

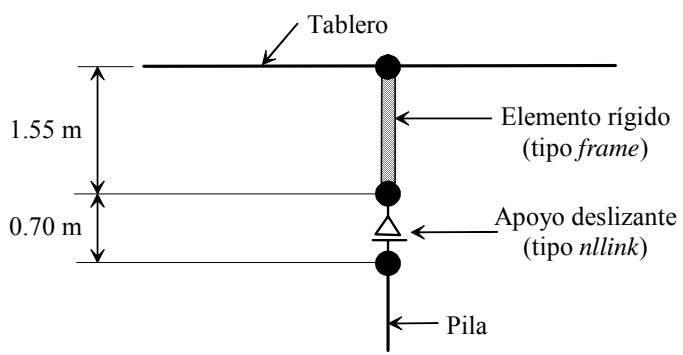

Figura 4. Modelación de cabezales de pilas con apoyos deslizantes

La superestructura se modeló por medio de elementos prismáticos frame. Como se consideró un tablero de concreto presforzado, se utilizaron las propiedades geométricas de la sección bruta, ya que el agrietamiento resulta despreciable. Además de los nudos en las intersecciones con las pilas y en los apoyos sobre los estribos, se colocó un nudo intermedio en cada tramo del tablero por la misma razón considerada en las pilas con altura mayor que $20 \mathrm{~m}$. Para modelar el arco se emplearon dos tipos de elementos frame; en las zonas adyacentes a los arranques se emplearon elementos no prismáticos con peralte variable, partiendo de $7 \mathrm{~m}$ en los arranques, y decreciendo linealmente hasta $5.5 \mathrm{~m}$ a una distancia de $60 \mathrm{~m}$ de los arranques, medidos horizontalmente (figura 1), mientras que en el resto del arco (zona central), se emplearon elementos prismáticos. Dada la elevada fuerza de compresión que la carga permanente induce al arco, se utilizaron las propiedades geométricas de la sección bruta. Además de los nudos en las intersecciones con las pilas y en los arranques, se consideraron tres nudos intermedios para poder captar la respuesta en diversos puntos del arco.

En otro orden de ideas, la filosofía de las normas internacionales de diseño sísmico de puentes, establece en general, que tras la ocurrencia del evento sísmico de proyecto, el puente debe mantener su integridad estructural y una adecuada resistencia residual, aunque ciertas partes del puente queden fuertemente dañadas, formándose articulaciones plásticas por flexión. Generalmente no es práctico ni deseable que se formen articulaciones plásticas en la superestructura, por lo que los extremos de las pilas suelen escogerse como los sitios para experimentar deformaciones inelásticas; para asegurar un comportamiento dúctil a flexión, es esencial que se inhiban los modos de falla frágiles. Así pues, el comportamiento inelástico del modelo se tomó en cuenta mediante articulaciones plásticas por flexión en la subestructura: extremos de pilas (empotradas en la cimentación o continuas con el arco o el tablero) y arranques 
del arco. Las relaciones momento-curvatura en dichos elementos se obtuvieron siguiendo los criterios de la norma española EHE (MF, 2000). Para obtenerlas se empleó el valor de la fuerza axial debida a carga permanente actuante en cada elemento y no se consideró ningún tipo de sobrerresistencia ni el efecto del confinamiento generado por el refuerzo transversal. La longitud de las articulaciones plásticas, zona en la que se considera que ocurre una curvatura plástica constante, se calculó de acuerdo con el criterio de la normativa japonesa (JRA, 1996), que establece que dicha longitud es función del claro de cortante. En la figura 5 se grafica la propuesta de la norma, donde $\mathrm{L}_{\mathrm{p}}$ es la longitud de la articulación plástica, $\mathrm{M} \mathrm{y} \mathrm{V}$ el momento flexionante y la fuerza cortante en la sección considerada, y d el peralte del elemento estructural en la dirección de análisis. La longitud de la articulación plástica es función del tipo probable de falla: cortante $(\mathrm{M} / \mathrm{Vd}<1)$, cortante-flexión $(1 \leq \mathrm{M} / \mathrm{Vd} \leq 3)$ y flexión $(\mathrm{M} / \mathrm{Vd}>3)$.

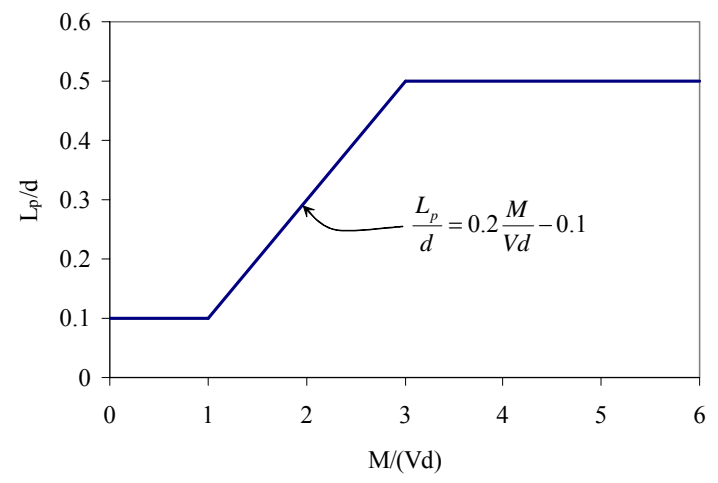

Figura 5. Longitud de articulaciones plásticas (JRA, 1996)

Contando con las relaciones momento-curvatura (M- $\phi$ ) y las longitudes de las articulaciones plásticas, se obtuvieron las relaciones momento-rotación (M- $\theta)$ de dichas articulaciones adoptando el modelo de plasticidad mostrado en la figura 6. Para modelar este comportamiento, en SAP2000, se recurre a elementos nllink, en particular al plastic 1. Así, se calcularon los factores de ductilidad rotacional $\mu_{\theta}=\theta_{\mathrm{u}} / \theta_{\mathrm{y}}$ (figura 6) para cada elemento, tanto en el plano como fuera de él. En este estudio se emplea la ductilidad rotacional $\mu_{\theta}=\theta_{\mathrm{u}} / \theta_{\mathrm{y}}$ de las articulaciones plásticas como medida de la capacidad de deformación de la estructura.

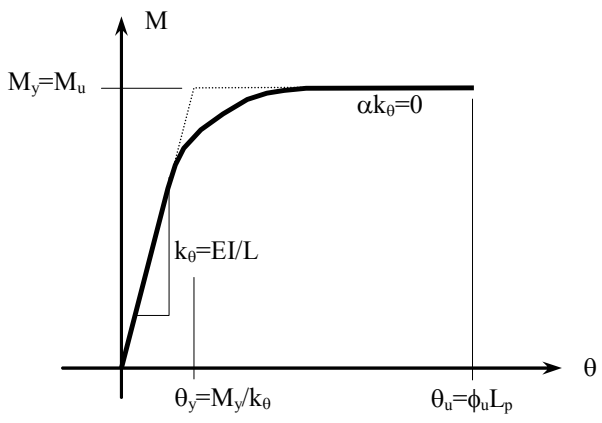

Figura 6. Diagrama momento-rotación de articulaciones plásticas 


\section{PROPIEDADES DINÁMICAS}

Para tener un primer acercamiento al comportamiento del modelo en estudio, se calcularon sus periodos y configuraciones modales, tomando en cuenta el efecto P- $\Delta$. En la figura 7 se presentan los periodos y el porcentaje de masa modal acumulada en las tres direcciones de análisis. El primer modo correspondió al periodo fundamental de la estructura fuera del plano y resultó de $3.811 \mathrm{~s}$, con una masa efectiva de $60.3 \%$. El segundo modo es el modo fundamental en el plano con una masa efectiva de $54.8 \%$ en dirección longitudinal, y el noveno modo resultó el modo predominante en dirección vertical con $37.4 \%$ de masa efectiva. En la figura 8 se muestran las configuraciones modales de estos tres modos predominantes (primero, segundo y noveno modos).

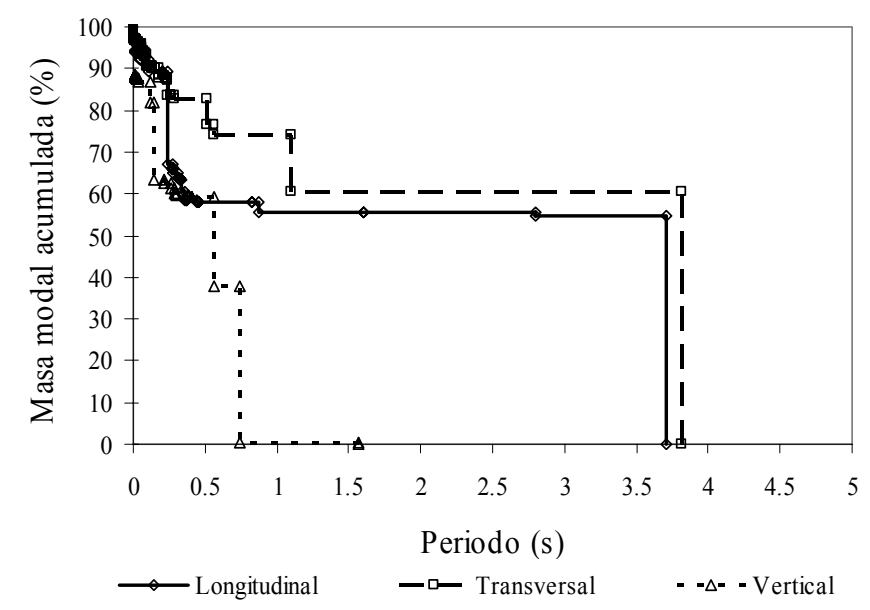

Figura 7. Periodos naturales y su correspondiente masa modal acumulada
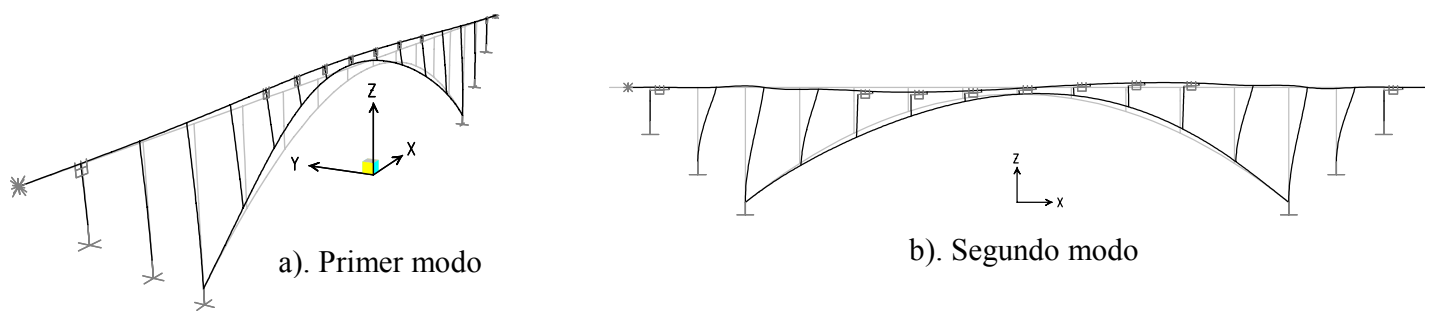

b). Segundo modo

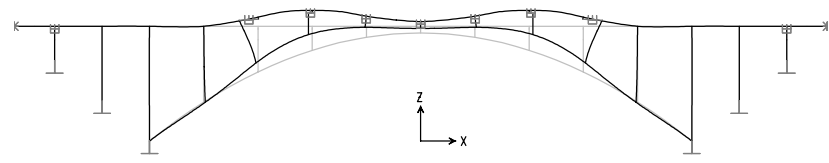

c). Noveno modo

Figura 8. Configuraciones modales de los modos predominantes: a) dirección transversal; $b$ ) dirección longitudinal; y c) dirección vertical 


\section{MOVIMIENTOS SÍSMICOS DEL TERRENO}

Para llevar a cabo la simulación numérica, se seleccionaron 10 registros de movimientos sísmicos del terreno de tres componentes cada uno. Se trata de registros obtenidos en roca o terreno duro, donde generalmente se construyen este tipo de estructuras y que corresponden a los sismos de Campano-Lucano (Italia, 1980), Michoacán (México, 1985), Northridge (EUA, 1994), Kobe (Japón, 1995) y Chi-Chi (Taiwán, 1999). Se seleccionaron dos estaciones de cada evento con la finalidad de contar con una variedad de movimientos con diferente duración y contenido de frecuencias. Los datos principales de estos registros aparecen en la tabla 1. Para efectos de presentación, las componentes se identifican con las letras L (longitudinal), $\mathrm{T}$ (transversal) y $\mathrm{V}$ (vertical).

Tabla 1. Movimientos sísmicos del terreno

\begin{tabular}{|c|c|c|c|c|c|c|c|}
\hline \multirow{2}{*}{ Sismo } & \multirow{2}{*}{$\mathrm{M}_{\mathrm{w}}$} & \multirow{2}{*}{ Estación } & \multirow{2}{*}{ Dirección } & \multicolumn{2}{|c|}{ Original } & \multicolumn{2}{|c|}{ Escalado ${ }^{*}$} \\
\hline & & & & $a_{\max }(g)$ & $V_{\max }(\mathrm{m} / \mathrm{s})$ & $a_{\max }(g)$ & $V_{\max }(\mathrm{m} / \mathrm{s})$ \\
\hline \multirow{6}{*}{$\begin{array}{l}\text { Campano- } \\
\text { Lucano, } \\
\text { Italia, } 1980\end{array}$} & \multirow{6}{*}{6.5} & \multirow{3}{*}{ Sturno } & $\mathrm{L}$ & 0.323 & 0.554 & 0.500 & 0.858 \\
\hline & & & $\mathrm{T}$ & 0.216 & 0.331 & 0.334 & 0.512 \\
\hline & & & $\mathrm{V}$ & 0.235 & 0.199 & 0.364 & 0.308 \\
\hline & & \multirow{3}{*}{$\begin{array}{c}\text { Torre del } \\
\text { Greco }\end{array}$} & $\mathrm{L}$ & 0.060 & 0.054 & 0.500 & 0.450 \\
\hline & & & $\mathrm{T}$ & 0.041 & 0.048 & 0.342 & 0.400 \\
\hline & & & V & 0.036 & 0.052 & 0.300 & 0.433 \\
\hline \multirow{6}{*}{$\begin{array}{l}\text { Michoacán, } \\
\text { México, } 1985\end{array}$} & \multirow{6}{*}{8.0} & \multirow{3}{*}{$\begin{array}{l}\text { Caleta de } \\
\text { Campos }\end{array}$} & $\mathrm{L}$ & 0.143 & 0.192 & 0.500 & 0.671 \\
\hline & & & $\mathrm{T}$ & 0.142 & 0.246 & 0.497 & 0.860 \\
\hline & & & V & 0.090 & 0.165 & 0.315 & 0.577 \\
\hline & & \multirow{3}{*}{$\begin{array}{c}\text { Mesa } \\
\text { Vibradora }\end{array}$} & $\mathrm{L}$ & 0.039 & 0.110 & 0.500 & 1.410 \\
\hline & & & $\mathrm{T}$ & 0.036 & 0.110 & 0.462 & 1.410 \\
\hline & & & V & 0.020 & 0.091 & 0.256 & 1.167 \\
\hline \multirow{6}{*}{$\begin{array}{l}\text { Northridge, } \\
\text { E. U. A., } 1994\end{array}$} & \multirow{6}{*}{6.7} & \multirow{3}{*}{$\begin{array}{l}\text { Castaic-Old } \\
\text { Ridge Route }\end{array}$} & $\mathrm{L}$ & 0.568 & 0.521 & 0.500 & 0.459 \\
\hline & & & $\mathrm{T}$ & 0.514 & 0.522 & 0.452 & 0.460 \\
\hline & & & $\mathrm{V}$ & 0.217 & 0.124 & 0.191 & 0.109 \\
\hline & & \multirow{3}{*}{$\begin{array}{l}\text { Riverside- } \\
\text { Airport }\end{array}$} & $\mathrm{L}$ & 0.064 & 0.031 & 0.500 & 0.242 \\
\hline & & & $\mathrm{T}$ & 0.059 & 0.027 & 0.461 & 0.211 \\
\hline & & & V & 0.022 & 0.023 & 0.172 & 0.180 \\
\hline \multirow{6}{*}{$\begin{array}{c}\text { Kobe, Japón, } \\
1995\end{array}$} & \multirow{6}{*}{6.9} & \multirow{3}{*}{ KJMA } & $\mathrm{L}$ & 0.821 & 0.813 & 0.500 & 0.495 \\
\hline & & & $\mathrm{T}$ & 0.599 & 0.743 & 0.365 & 0.452 \\
\hline & & & V & 0.343 & 0.383 & 0.209 & 0.233 \\
\hline & & \multirow{3}{*}{ OKA } & $\mathrm{L}$ & 0.081 & 0.048 & 0.500 & 0.296 \\
\hline & & & $\mathrm{T}$ & 0.059 & 0.032 & 0.364 & 0.198 \\
\hline & & & V & 0.038 & 0.025 & 0.235 & 0.154 \\
\hline \multirow{6}{*}{$\begin{array}{c}\text { Chi-Chi, } \\
\text { Taiwán, } 1999\end{array}$} & \multirow{6}{*}{7.6} & \multirow{3}{*}{ TCU052 } & $\mathrm{L}$ & 0.419 & 1.184 & 0.500 & 1.413 \\
\hline & & & $\mathrm{T}$ & 0.348 & 1.590 & 0.415 & 1.897 \\
\hline & & & V & 0.241 & 1.105 & 0.288 & 1.319 \\
\hline & & \multirow{3}{*}{ TCU014 } & $\mathrm{L}$ & 0.075 & 0.135 & 0.500 & 0.900 \\
\hline & & & $\mathrm{T}$ & 0.058 & 0.242 & 0.387 & 1.613 \\
\hline & & & $\mathrm{V}$ & 0.018 & 0.062 & 0.120 & 0.413 \\
\hline
\end{tabular}

* Escalados a $a_{\max }=0.5 \mathrm{~g}$

A pesar de que la velocidad del terreno puede ser un mejor indicador del potencial destructor de un sismo en estructuras con periodo largo (Tso et al, 1992), la práctica más común adoptada por las normas internacionales sigue siendo usar la aceleración máxima del terreno $\left(a_{\max }\right)$ como medida de la intensidad del movimiento. Es por esto, que para evaluar la respuesta 
sísmica del modelo estructural que se estudia en este trabajo, las componentes originales de aceleración se escalaron linealmente de tal manera que la $a_{\max }$ en dirección longitudinal fuese igual a $0.5 \mathrm{~g}$ para cada estación; éste es un valor representativo de la $a_{\max }$ en suelo duro en regiones con alta actividad sísmica, como lo son la zona de subducción en la costa del Pacífico mexicano (CFE, 1993), varias zonas de la costa de California (AASHTO, 1994), y algunas regiones de Japón (JRA, 1996). En la misma tabla 1 se presentan los valores máximos de aceleración $\left(a_{\max }\right)$ y velocidad $\left(v_{\max }\right)$ del terreno de los registros originales y de los escalados. Cada conjunto de tres componentes de los movimientos sísmicos escalados se aplicó al modelo del puente.
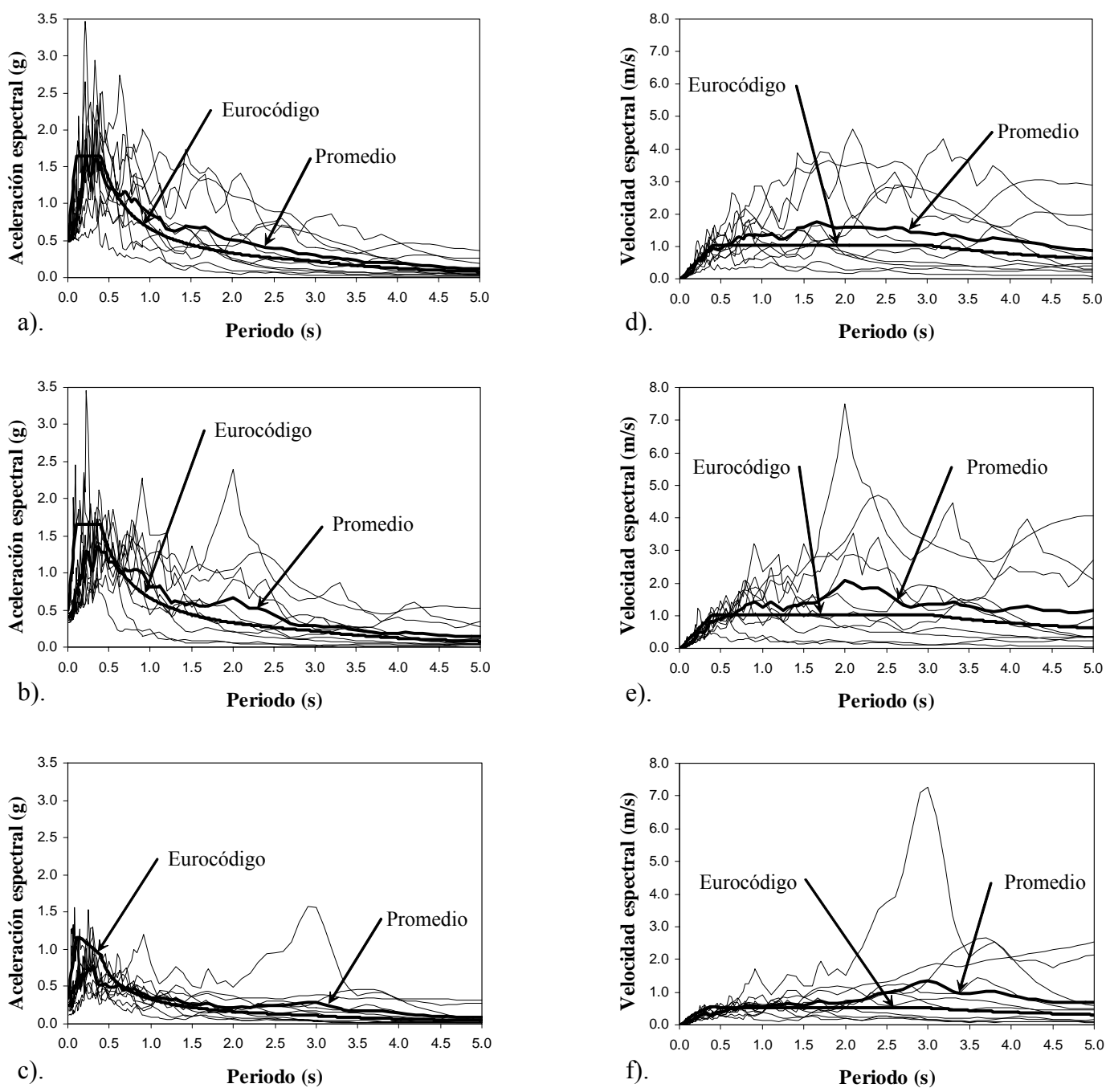

Figura 9. Espectros de respuesta ( $\xi=2 \%$ ) de los registros escalados: $a), b$ ) y c), aceleraciones longitudinal, transversal y vertical; d), e) y f), velocidades longitudinal, transversal y vertical 
En la figura 9 se presentan para los 10 movimientos y sus tres componentes, los espectros de respuesta de pseudoaceleración y pseudovelocidad para un porcentaje de amortiguamiento crítico $\xi=2 \%$. También se muestra con fines comparativos, el espectro de diseño del Eurocódigo $8(\xi=2 \%)$ para una $a_{\max }=0.5 \mathrm{~g}$ en las direcciones horizontales y $a_{\max }=0.35 \mathrm{~g}$ en dirección vertical; se presentan los espectros del Eurocódigo, ya que con éste se diseñó el puente. Como se observa, aproximadamente la mitad de los espectros excede al espectro del código en la región de periodos largos. Más aún, el espectro promedio también excede al del código en la misma región.

\section{ANÁLISIS DINÁMICO}

Cada análisis dinámico transitorio no lineal constó de dos etapas. En la primera etapa se aplicó gradualmente la carga permanente, partiendo de condiciones iniciales nulas, en incrementos de 0.01 segundos hasta completar el $100 \%$ de esta carga en un tiempo de 20 segundos (más de 5 veces el periodo del modo fundamental) y manteniéndola durante 20 segundos más para estabilizar el movimiento; para eliminar cualquier vibración debida a la aplicación de la carga permanente, en dicha etapa se usó un amortiguamiento modal de $99.9 \%$. En una segunda fase, partiendo del estado de esfuerzos y deformaciones inducido por la aplicación previa de la carga permanente, se aplicaron los acelerogramas a los apoyos de la estructura también en incrementos de 0.01 segundos, con un amortiguamiento modal de $2 \%$, valor recomendado por las normas AASHTO (1994) para puentes de concreto. Para cada análisis dinámico se emplearon los primeros 100 modos asociados a sus correspondientes vectores de Ritz, incluyendo los de los elementos con comportamiento no lineal; se usaron sólo los primeros 100 modos, ya que con ellos se alcanzaba más del $90 \%$ de la masa modal acumulada. Se consideró una excitación uniforme en todos los apoyos del puente (movimiento sincrónico de la cimentación). Los análisis se continuaron durante 10 segundos después de terminar el movimiento del suelo, con la finalidad de observar la vibración libre del modelo durante este tiempo. En la figura 10 se representa gráficamente el proceso descrito, donde $t$ es el tiempo, $W$ la carga permanente y $a$ la aceleración en los apoyos del modelo.

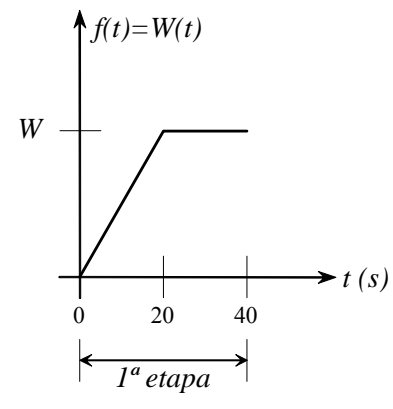

a). Carga permanente

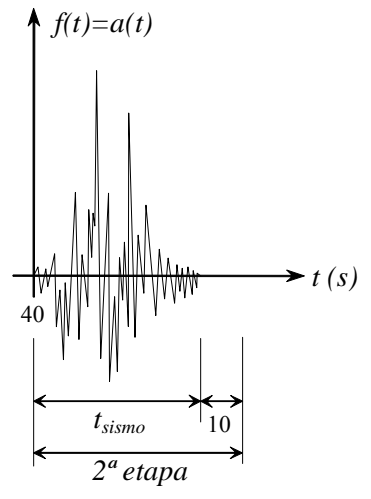

b). Movimiento sísmico

Figura 10. Proceso de análisis dinámico 


\section{COMPORTAMIENTO DEL MODELO}

Para la evaluación de las acciones gravitacionales se consideró el peso propio de la estructura más una carga permanente de $2.5 \mathrm{kN} / \mathrm{m}^{2}$ actuando sobre la superficie del tablero. En la figura 11 se presenta un resumen de las fuerzas internas en el arco. La coordenada $X=0$ corresponde a la clave del arco, mientras que $X=-200$ y $X=200$ corresponden a los arranques. En el inciso a) de esta figura se muestra la variación de la fuerza normal en función de dicha coordenada. La compresión máxima de $353.05 \mathrm{MN}$ en los arranques, representa el 35\% de la carga de rotura por compresión, de acuerdo con la norma EHE (MF, 2000). En el inciso b) de la misma figura se señala, para el plano del modelo, la variación del momento flexionante a lo largo de la directriz del arco, así como la del momento último, calculado éste a partir de la fuerza axial producida por la carga permanente, siguiendo también la norma EHE; el momento flexionante originado por la carga permanente representa apenas un $15 \%$ del momento último en el caso más desfavorable, por lo que la capacidad remanente queda disponible para absorber los efectos sísmicos.

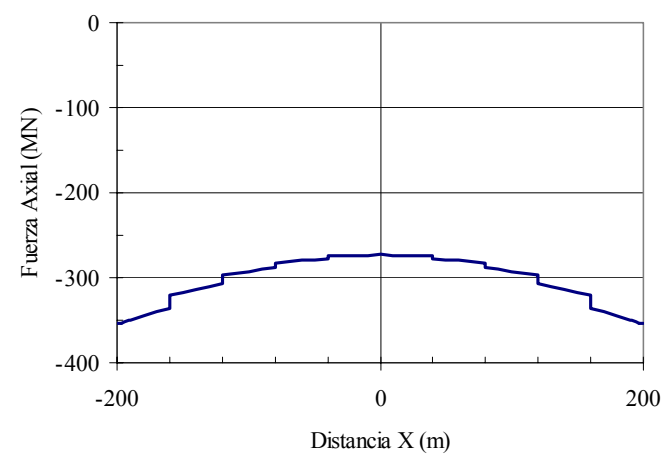

a). Fuerza normal

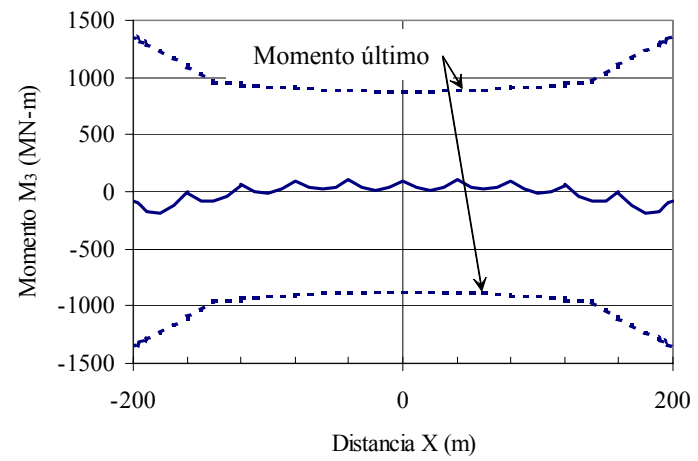

b). Momento flexionante en el plano

Figura 11. Fuerzas internas en el arco debidas a acciones permanentes

Para evaluar la respuesta sísmica del modelo, cada conjunto de tres componentes de los movimientos escalados descritos con anterioridad (tabla 1) se aplicó al modelo. La componente L se aplicó en la dirección longitudinal del modelo (X), la $\mathrm{T}$ en dirección transversal (Y), y la $\mathrm{V}$ en dirección vertical $(Z)$. De un análisis dinámico transitorio no lineal se obtiene una gran cantidad de información, por lo que es importante identificar relativamente pocos parámetros que representen el comportamiento global de la estructura. Para interpretar los resultados de los diferentes análisis se estudiaron los desplazamientos en el centro del claro de la estructura, y el comportamiento no lineal de los arranques del arco, de los extremos de las pilas principales P3 y P13, y del extremo inferior de las pilas P2, P4, P12 y P14. Asimismo, se determinaron la energía de entrada y la disipada por amortiguamiento modal y por amortiguamiento histerético.

En la figura 12 se presentan las historias de desplazamiento en el centro del claro del modelo ante el evento de Chi-Chi, estación TCU052, uno de los que originaron las mayores respuestas del modelo. En dirección longitudinal se grafican por separado las historias temporales tanto del tablero como de la clave del arco, dado que la conectividad que existe entre tablero y 
arco permite el deslizamiento relativo entre ambos. En las direcciones transversal y vertical sólo se muestra una sola curva, ya que los desplazamientos del tablero y de la clave del arco son prácticamente iguales en su sección media. En el inciso a) de la figura se presentan los desplazamientos en dirección longitudinal; los movimientos entre el tablero y la clave del arco resultaron notoriamente diferentes. Al final del movimiento, el tablero quedó con un desplazamiento residual longitudinal del orden de $0.6 \mathrm{~m}$, evidenciando daño estructural en los extremos inferiores de las pilas $\mathrm{P} 2$, P4, P12, y P14, y en ambos extremos de las pila P3 y P13, que son las encargadas de resistir las fuerzas de inercia de la superestructura. Para las pilas P2 y $\mathrm{P} 14$, de $55.25 \mathrm{~m}$ de altura, este desplazamiento residual representa un desplome de $0.62^{\circ}$; desplomes mayores que un grado fueron causa suficiente para demoler 88 pilas del Viaducto Hanshin durante el sismo de Kobe en 1995 (Fujino et al, 2005). Por otro lado, la clave del arco no experimentó desplazamiento permanente. El inciso b) de la misma figura muestra los desplazamientos en dirección transversal; también se observa un desplazamiento residual importante de aproximadamente $0.9 \mathrm{~m}$, producto de la deformación inelástica de los arranques del arco y de las bases de las pilas que soportan las fuerzas de inercia de la superestructura, además de las propias, en esta dirección. En el inciso c) se presenta la historia de desplazamientos verticales; el movimiento comienza con un desplazamiento vertical descendente de $0.178 \mathrm{~m}$, producto de la carga permanente, desarrollándose con oscilaciones de poca amplitud.

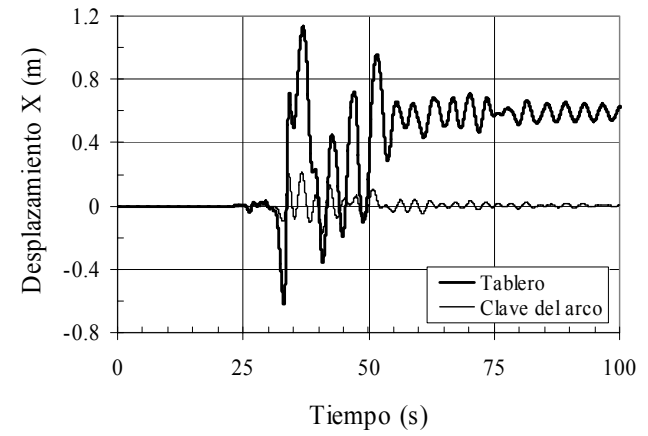

a). Desplazamientos longitudinales

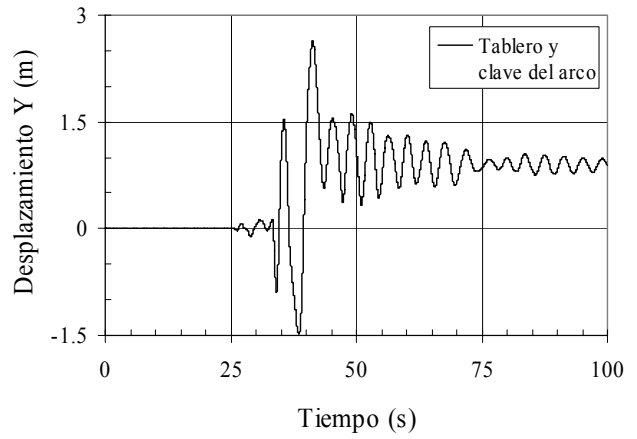

b). Desplazamientos transversales

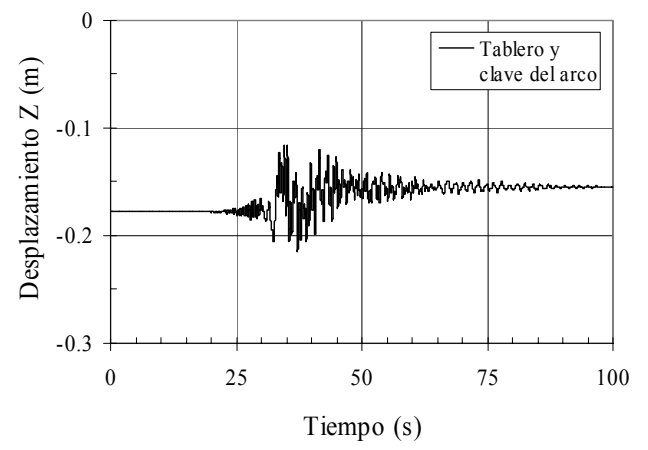

c). Desplazamientos verticales

Figura 12. Historias de desplazamiento en el centro del claro, sismo de Chi-Chi, estación TCU052 
En la tabla 2 se presentan los desplazamientos máximos del modelo originados por los 10 conjuntos de registros sísmicos. En dicha tabla se pueden identificar ya algunas tendencias generales. De los 10 eventos, el de la estación Mesa Vibradora causó los mayores desplazamientos en direcciones longitudinal y vertical. El evento de la estación TCU052 originó el mayor desplazamiento transversal. Los sismos de Northridge y de Kobe causaron los menores desplazamientos. En la última línea de la tabla se muestran las medias aritméticas de los desplazamientos. Nótese que en las direcciones longitudinal y transversal, las medias del tablero, de $0.861 \mathrm{~m}$ y $0.815 \mathrm{~m}$ respectivamente, fueron excedidas por los eventos de Mesa Vibradora, TCU052 y TCU014, que son los movimientos con mayores $v_{\text {máx }}$ (tabla 1, última columna).

Tabla 2. Desplazamientos máximos en el centro el claro

\begin{tabular}{|c|c|c|c|c|c|}
\hline \multirow{2}{*}{ Sismo } & Estación & $\begin{array}{c}\text { Longitudinal } \\
\text { Tablero }(\mathrm{m})\end{array}$ & $\begin{array}{c}\text { Longitudinal } \\
\text { Arco }(\mathrm{m})\end{array}$ & $\begin{array}{c}\text { Transversal } \\
\text { Tablero }(\mathrm{m})\end{array}$ & $\begin{array}{c}\text { Vertical } \\
\text { Tablero }(\mathrm{m})\end{array}$ \\
\hline \multirow{2}{*}{ Campano-Lucano } & Sturno & 0.772 & 0.196 & 0.650 & 0.214 \\
\cline { 2 - 6 } & Torre del Greco & 0.540 & 0.087 & 0.713 & 0.215 \\
\hline \multirow{2}{*}{ Michoacán } & Caleta de Campos & 0.308 & 0.070 & 0.641 & 0.210 \\
\cline { 2 - 6 } & Mesa Vibradora & 3.650 & 0.227 & 1.231 & 0.251 \\
\hline \multirow{2}{*}{ Northridge } & Castaic - ORR & 0.201 & 0.042 & 0.299 & 0.188 \\
\cline { 2 - 6 } & Riverside - Airport & 0.064 & 0.033 & 0.083 & 0.194 \\
\hline \multirow{2}{*}{ Kobe } & KJMA & 0.208 & 0.044 & 0.344 & 0.215 \\
\cline { 2 - 6 } & OKA & 0.167 & 0.028 & 0.184 & 0.197 \\
\hline \multirow{2}{*}{ Chi-Chi } & TCU052 & 1.133 & 0.209 & 2.643 & 0.214 \\
\cline { 2 - 6 } & TCU014 & 1.562 & 0.167 & 1.363 & 0.210 \\
\hline Media & & $\mathbf{0 . 8 6 1}$ & $\mathbf{0 . 1 1 0}$ & $\mathbf{0 . 8 1 5}$ & $\mathbf{0 . 2 1 1}$ \\
\hline
\end{tabular}

Para mostrar el comportamiento del arco, elemento principal de la estructura en estudio, en la figura 13 se presenta la respuesta del arranque izquierdo originada por el sismo de Chi-Chi, estación TCU052. En el inciso a) de la figura 13 se muestra la historia de fluctuación de la fuerza axial, que se inicia con una compresión de $353.05 \mathrm{MN}$, producto de la aplicación previa de la carga permanente. La compresión en este arranque llegó a ser $43 \%$ mayor y $47 \%$ menor que la originada por la carga permanente para un instante dado. En el inciso b) se presenta el diagrama momento-rotación del arranque izquierdo correspondiente a flexión transversal del puente; se observa un marcado comportamiento no lineal de este elemento, presentándose rotaciones residuales asociadas con deformaciones permanentes de la estructura fuera del plano. En el inciso c) se presenta un diagrama momento-rotación en el plano del puente, donde se muestra que se alcanzó el momento plástico, acompañado de rotaciones moderadas.

En la figura 14 se señala un resumen de la respuesta de los elementos con comportamiento no lineal en términos de la relación demanda media/capacidad rotacional. Como se mencionó con anterioridad, aquí se emplea la ductilidad rotacional $\mu_{\theta}=\theta_{\mathrm{u}} / \theta_{\mathrm{y}}$ como medida de la capacidad de deformación de la estructura. Como se observa en la figura, para flexión fuera del plano los arranques del arco vieron excedida su capacidad rotacional en $28 \%$, mientras que las demandas medias en los extremos de las pilas estuvieron por debajo de su capacidad rotacional. Por el contrario, para flexión en el plano, la demanda media solamente representa el $24 \%$ de la capacidad rotacional de los arranques, mientras que los extremos de las pilas vieron rebasada por amplio margen su capacidad rotacional: $54 \%$ y $46 \%$ en los extremos 
inferior y superior, respectivamente, de las pilas P3 y P13, 115\% en el extremo inferior de las P2 y P14, y 132\% en el extremo inferior de las P4 y P12.

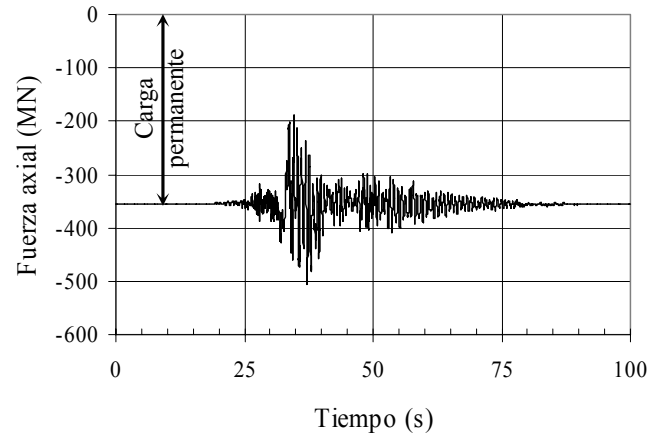

a). Fluctuación de la fuerza axial

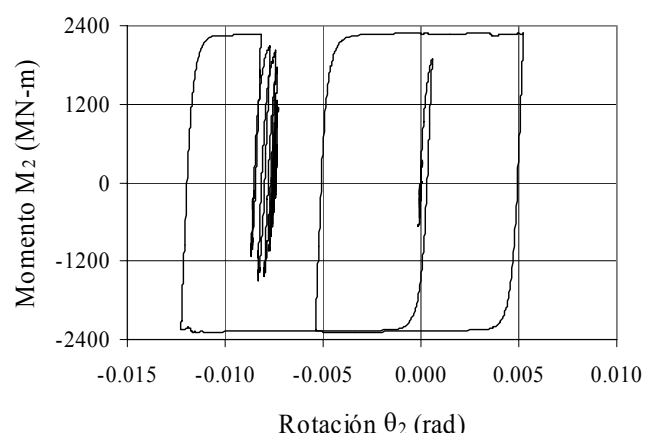

b). Diagrama M- $\theta$ fuera del plano

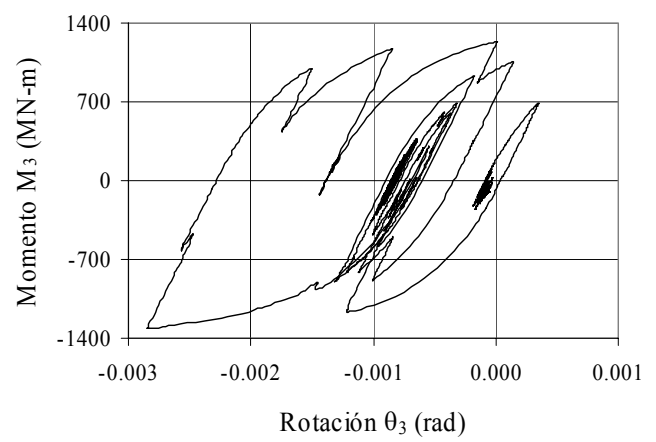

c). Diagrama M- $\theta$ en el plano

Figura 13. Respuesta del arranque izquierdo del arco ante el sismo de Chi-Chi, estación TCU052

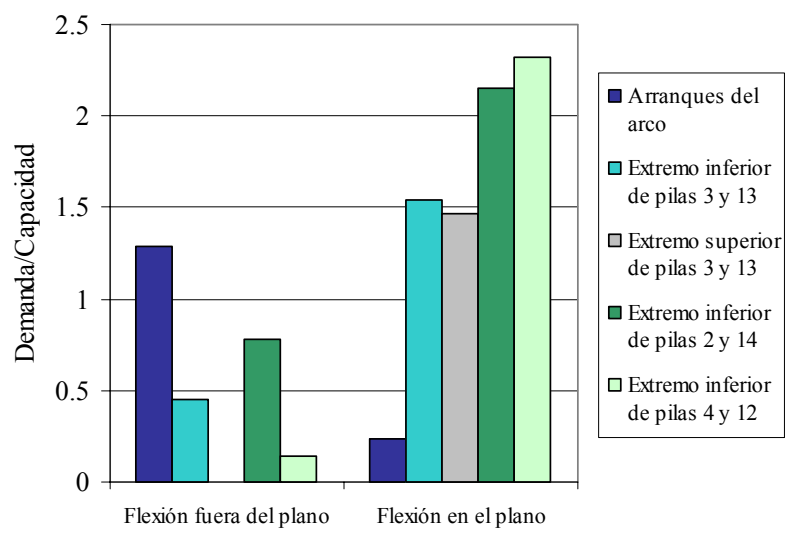

Figura 14. Relaciones demanda media/capacidad rotacional en la subestructura del modelo 
Es posible establecer cualitativamente el nivel de daño que puede sufrir la estructura en estudio ante un determinado evento sísmico, si se comparan la energía disipada por amortiguamiento modal y por amortiguamiento histerético con la energía de entrada. En la figura 15 se presentan, en términos de la energía de entrada, los porcentajes de las energías modal e histerética. Se aprecia que los sismos con mayor energía histerética son los de las estaciones Mesa Vibradora, TCU052 y TCU014, con 71\%, 80\% y 74\%, respectivamente, que además son los eventos con mayores $v_{\text {máx }}$ (tabla 1, última columna). Cabe hacer notar que no se evaluó la energía disipada por la cimentación ni por los apoyos, lo cual reduciría la energía histerética.

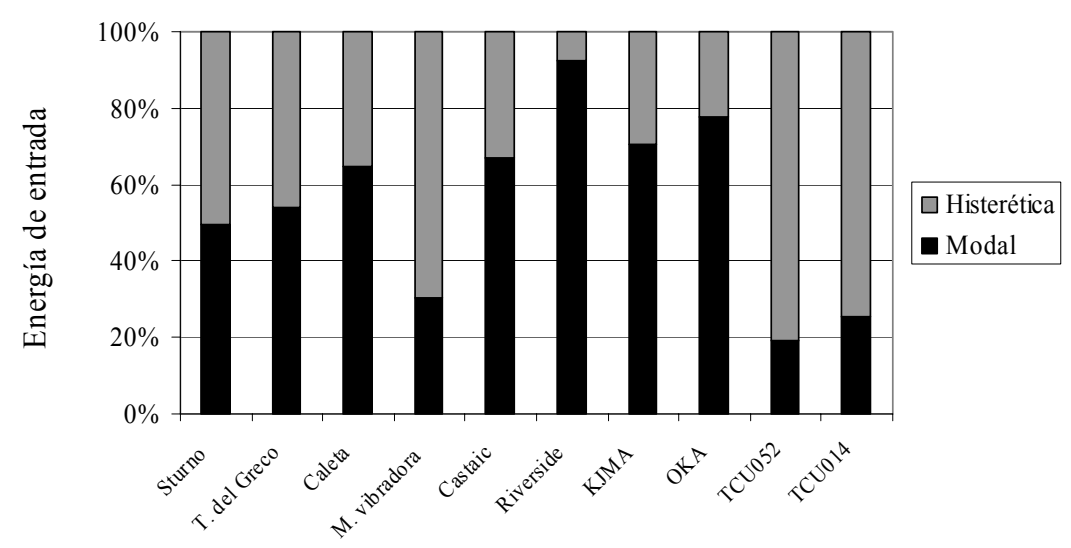

Figura 15. Distribuciones de energía acumulada modal e histerética

\section{EVALUACIÓN DE LA RESPUESTA}

\section{Comportamiento global}

Se evaluó la respuesta del modelo sometiéndolo a diez conjuntos de registros sísmicos con tres componentes cada uno; algunos de ellos, causaron un marcado comportamiento no lineal a la subestructura, en particular los de mayores $v_{\text {máx }}$. En la figura 16 se ilustran los elementos en los que las demandas de ductilidad excedieron la capacidad o ductilidad rotacional. Para flexión fuera del plano los arranques izquierdo y derecho del arco vieron excedida su capacidad rotacional en $29 \%$ y $27 \%$ respectivamente, mientras que las demandas medias en los extremos de las pilas estuvieron por debajo de su capacidad rotacional. Por el contrario, para flexión en el plano no se observó daño significativo en los arranques, mientras que los extremos de las pilas sí vieron rebasada por amplio margen su capacidad rotacional, desde $42 \%$ en el extremo superior de la pila P13, hasta $143 \%$ en el extremo inferior de la pila P4, poniendo de manifiesto la conveniencia de reducir la respuesta del modelo en el plano. En este sentido, resulta conveniente explorar la reducción de la respuesta empleando otro arreglo de conectividades entre superestructura y subestructura, o bien, usando algún sistema de control pasivo, como por 
ejemplo, amortiguadores viscosos; esta segunda alternativa está siendo investigada por los autores.

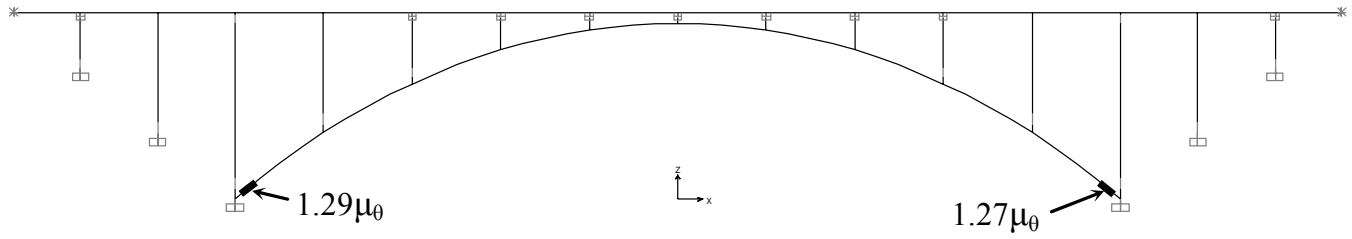

a). Flexión fuera del plano

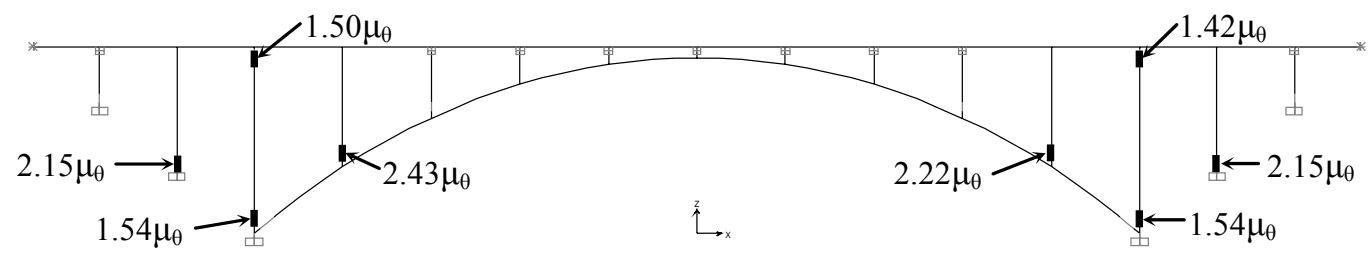

b). Flexión en el plano

Figura 16. Elementos con daño excesivo por sismo

\section{Movimientos sísmicos}

Durante el desarrollo de este trabajo quedó de manifiesto que los eventos de las estaciones Mesa Vibradora, TCU052 y TCU014, produjeron las mayores respuestas del modelo. Estos sismos se caracterizan por contar con las mayores velocidades máximas del terreno $\left(v_{\text {máx }}\right)$, como se observa en la tabla 1. Así pues, para predecir la respuesta de sistemas estructurales con periodo largo como el que nos ocupa, debe tenerse en cuenta la $v_{\text {máx }}$ como medida del potencial destructor del sismo.

Pese a lo anterior, la práctica más común adoptada por normas de diseño sísmico sigue siendo describir la intensidad del movimiento en términos de la aceleración máxima del terreno $\left(a_{\text {máx }}\right)$, razón por la cual los registros originales se escalaron linealmente a una $a_{\text {máx }}$ de $0.5 \mathrm{~g}$. Sin embargo, escalar registros sísmicos en función de la $a_{\text {máx }}$ puede generar dispersiones importantes en la estimación de la respuesta no lineal de estructuras con periodo largo (Kurama y Farrow, 2003). Para explorar esta posibilidad, a continuación, se compara la respuesta del modelo en términos del desplazamiento longitudinal escalando los sismos por $a_{\text {máx }}$ y por $v_{\text {máx. }}$. En el primer caso, como se ha reiterado, todos los registros se escalaron a una $a_{\text {máx }}$ de $0.5 \mathrm{~g}$. El segundo caso se planteó de tal manera que los registros se escalasen a una misma $v_{\text {máx }}$ y que simultáneamente la media de las $a_{\text {máx }}$ fuese igual a $0.5 \mathrm{~g}$; ambas condiciones se satisfacen al escalar los sismos de la tabla 1 a una $v_{\text {máx }}$ de $0.523 \mathrm{~m} / \mathrm{s}$ en la dirección de mayor $a_{\text {máx. }}$ En la figura 17 se muestran los espectros medios de respuesta para ambos casos y se comparan con el del Eurocódigo 8; nótese que los tres tienen una misma $a_{\text {máx }}$ de $0.5 \mathrm{~g}$. 
En la tabla 3 se presentan la media y el coeficiente de variación de los desplazamientos longitudinales normalizados del tablero sometiendo el modelo a los sismos escalados. Como se observa, los desplazamientos longitudinales presentan una media de 0.223 y un coeficiente de variación de 1.273 al escalar los sismos por aceleración, mientras que la media resulta de $0.497 \mathrm{y}$ el coeficiente de variación de 0.554 para los sismos escalados por velocidad. Ante estos resultados, es deseable que las normas de diseño sísmico incluyesen también de manera transparente el uso de la $v_{\text {máx }}$ como medida de la peligrosidad en puentes con periodos largos; este parámetro ha mostrado ser un mejor indicador del contenido de frecuencias y del potencial destructor de un sismo para estructuras de periodo largo (Tso et al, 1992). Otro método que puede ser atractivo para escalar sismos en casos similares al aquí presentado, es el de la máxima velocidad incremental (MVI). La MVI puede ser el mejor indicador del potencial destructor de un sismo que la $a_{m a ́ x}$, ya que capta las características impulsivas del movimiento (Kurama y Farrow, 2003). Sin embargo, actualmente no existen métodos aceptados para determinar la probabilidad de excedencia de una cierta MVI para un sitio dado; se requiere mayor investigación al respecto.

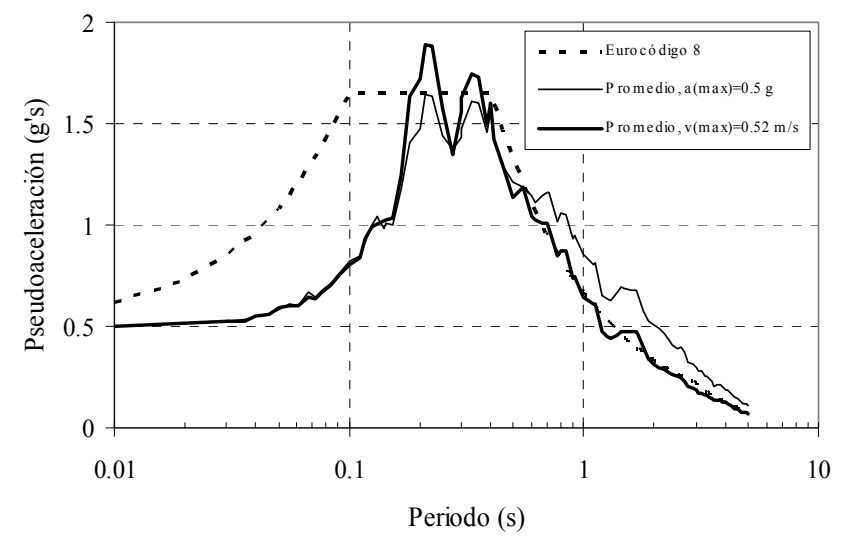

Figura 17. Espectros elásticos de respuesta de los registros escalados, $\xi=2 \%$, dirección más fuerte

Tabla 3. Desplazamientos máximos normalizados de la superestructura

\begin{tabular}{|c|c|c|}
\hline \multirow{2}{*}{ Sismos } & \multicolumn{2}{|c|}{ Desplazamiento longitudinal } \\
\cline { 2 - 3 } & Media & Coeficiente de variación \\
\hline $\begin{array}{c}\text { Escalados por aceleración, } \\
a_{\max }=0.5 \mathrm{~g}\end{array}$ & 0.233 & 1.273 \\
\hline $\begin{array}{c}\text { Escalados por velocidad, } \\
v_{\max }=0.52 \mathrm{~m} / \mathrm{s}\end{array}$ & 0.497 & 0.554 \\
\hline
\end{tabular}

\section{Fluctuación de la fuerza normal}

Como se ha manifestado con anterioridad, las relaciones momento-curvatura (M- $\phi$ ) de los elementos con comportamiento no lineal se obtuvieron considerando la fuerza axial producida 
por la carga permanente. Sin embargo, cuando se toma en cuenta la fluctuación de la carga axial durante el movimiento sísmico es de esperarse que ocurran cambios en las relaciones M- $\phi$. Durante la ocurrencia de un movimiento sísmico fuerte se presenta una variación importante de la fuerza axial en el arco, por lo que sería deseable considerar la interacción no lineal de dicha fuerza y el momento flexionante. Para evaluar de manera aproximada la influencia de esta variable en el comportamiento de la estructura en estudio, en particular en los arranques del arco, se recurrió a la siguiente metodología: 1) emplear inicialmente la relación $M-\phi$ derivada de la fuerza normal causada por la carga permanente y hacer un primer análisis; 2) reanalizar la estructura con las relaciones $M-\phi$ debidas a la fuerza axial mínima y máxima ocasionadas por la carga gravitacional más sismo. Cabe señalar que las fuerzas axiales mínima y máxima actúan en un sólo un instante, por lo que los resultados resultan conservadores.

En la figura 18 se muestran los intervalos de fluctuación de la compresión que cada sismo produce en ambos arranques del arco; estos valores se obtuvieron a partir de las relaciones M- $\phi$ obtenidas con la fuerza axial causada por la carga permanente. En la figura se ilustra también la magnitud de la compresión producida por la carga permanente $(353.05 \mathrm{MN})$, y la media de las fuerzas axiales mínima $(155.91 \mathrm{MN})$ y máxima $(541.17 \mathrm{MN})$ producidas por la carga permanente más sismo, lo que indica que la fuerza de compresión producida por la carga permanente en los arranques del arco experimenta una variación del orden de $\pm 50 \%$ durante la ocurrencia del movimiento sísmico.

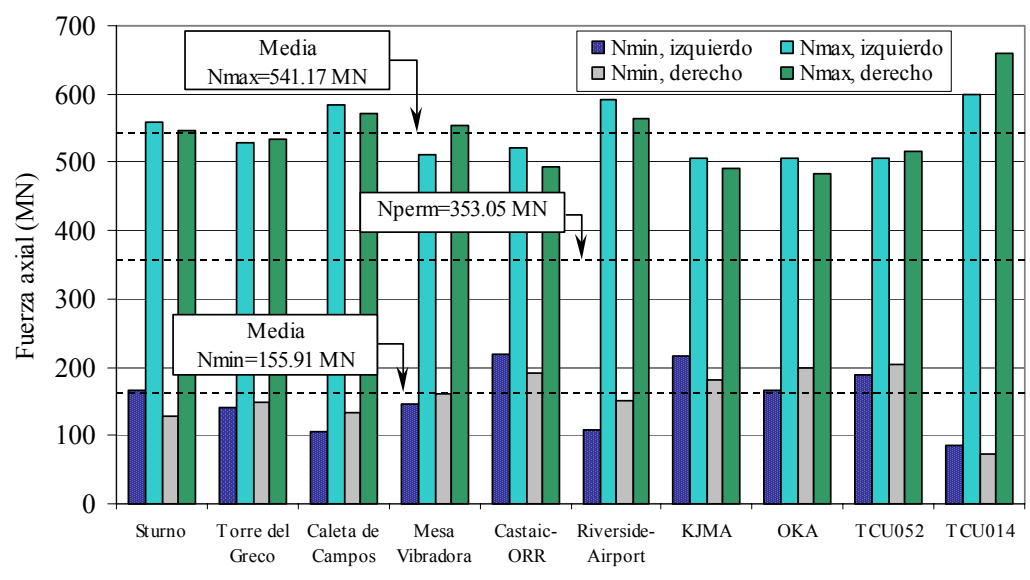

Figura 18. Fluctuación de la compresión en los arranques del arco

Con los valores mínimo y máximo de la fuerza normal se obtuvieron nuevas relaciones M- $\phi$ y M- $\theta$ para los arranques del arco. El modelo se reanalizó con estas nuevas propiedades que toman en cuenta de manera aproximada la fluctuación de la fuerza axial. En la figura 19 se muestran las demandas medias de ductilidad rotacional en ambos arranques para los tres niveles de fuerza axial y se comparan con sus capacidades rotacionales. Como se observa, en los tres casos la demanda resultó menor que la capacidad rotacional. La mayor demanda se presentó para el caso de compresión mínima, con una magnitud de 3.242; el caso más desfavorable resultó el de compresión máxima, con una demanda de 2.183 y una capacidad de 3.609. Estos resultados están 
referidos al caso de flexión en el plano del puente y reflejan la necesidad de tomar en cuenta el efecto de la fluctuación de la fuerza normal en el arco durante la ocurrencia de un movimiento sísmico intenso. Para el caso de flexión transversal cabe recordar que, como se expuso anteriormente, la demanda media de ductilidad ya había excedido la capacidad rotacional de los arranques del arco. Para tomar en cuenta el efecto de fluctuación de la fuerza normal también se puede recurrir a métodos más refinados, como por ejemplo, modelar la estructura mediante elementos finitos tipo fibra (Sakai y Kawashima, 2002).

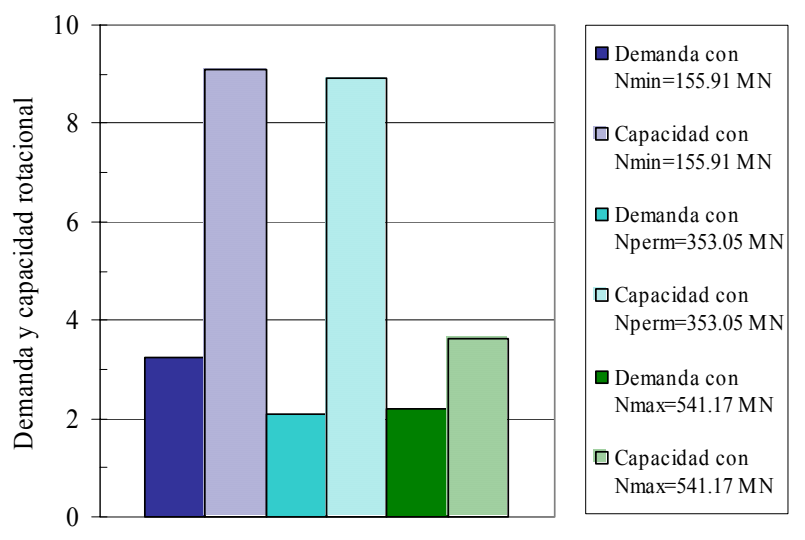

Figura 19. Demandas medias de ductilidad y capacidad rotacional de los arranques del arco para tres niveles de fuerza normal. Flexión en el plano del modelo

\section{Variabilidad espacial}

Los puentes en arco generalmente se construyen sobre terrenos rocosos, donde las ondas sísmicas viajan a altas velocidades, por lo que es razonable suponer que el movimiento del suelo es uniforme en toda la cimentación del puente. Los casos presentados se analizaron bajo esta consideración de excitación uniforme. Sin embargo, si existen discontinuidades geológicas o características topográficas capaces de introducir variaciones importantes en las características del movimiento del suelo o si la longitud del puente es muy grande, debe considerarse la variabilidad espacial del movimiento sísmico.

Para explorar la influencia de la variabilidad espacial en el modelo en estudio, se planteó el siguiente análisis que considera solamente el efecto del paso de onda. Este efecto no modifica la forma de los movimientos sísmicos en las diferentes ubicaciones de los apoyos del puente, y se incluye en los modelos de análisis sísmico aplicando retardos sistemáticos a las historias temporales. El programa SAP2000 permite modelar la excitación múltiple del terreno aplicando a los apoyos historias temporales de desplazamiento.

El modelo se sometió al sismo de Campano-Lucano, estación Sturno (tabla 1), aplicando a los apoyos del puente las historias de desplazamiento en las direcciones longitudinal, transversal 
y vertical resultantes de la segunda integración de los acelerogramas correspondientes. A los apoyos del lado derecho del puente (arranque derecho, pilas P13 a P15, y estribo E2, figura 1), se aplicaron las historias coherentes de desplazamiento con un retraso de un segundo respecto a los del lado izquierdo; considerando que la longitud total del modelo es de $600 \mathrm{~m}$, este retraso en el tiempo de llegada implica una velocidad de propagación de las ondas sísmicas de $600 \mathrm{~m} / \mathrm{s}$, que corresponde a subsuelo clase "A" según el Eurocódigo 8. Vale la pena señalar que el Eurocódigo 8 Parte 2 (CEN, 1998b) recomienda que se tenga en cuenta el efecto de la variabilidad espacial cuando la longitud del puente exceda de $600 \mathrm{~m}$ o cuando existan discontinuidades geológicas o características topográficas capaces de generar variaciones importantes en las características del movimiento del terreno.

Para comparar el comportamiento del modelo sometido a movimiento uniforme y a excitación múltiple, la respuesta se separó en dos componentes: una cuasi estática, causada por el movimiento asincrónico de los apoyos en cualquier instante, y otra debida a la vibración. Tanto en dirección longitudinal como en dirección transversal los desplazamientos máximos para el caso de excitación múltiple se redujeron a menos del 50\% de los correspondientes a movimiento uniforme; sin embargo, todo lo contrario ocurrió en dirección vertical. En la figura 20 se presentan las historias de desplazamiento vertical en el centro del claro debidas a la vibración; como se observa en la figura, los desplazamientos para el caso de excitación múltiple aumentaron considerablemente, hasta un máximo absoluto de $0.924 \mathrm{~m}$, como consecuencia de la mayor excitación de los modos verticales.

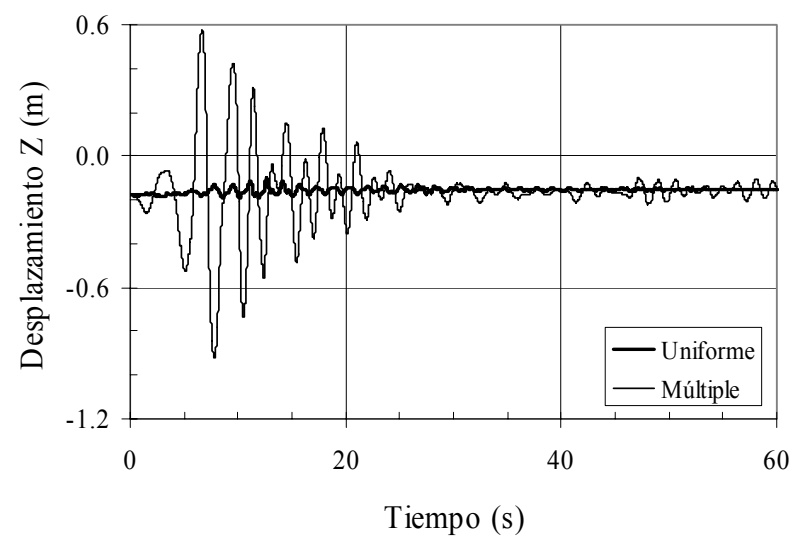

Figura 20. Historias de desplazamiento vertical en el centro del claro. Sismo de Campano-Lucano, estación Sturno

En la figura 21 se ilustra la respuesta de los arranques del arco en términos de fuerzas axiales y rotaciones, comparando el caso de variabilidad espacial (VE) con el de movimiento uniforme (MU). Estos parámetros normalizados incluyen tanto su valor cuasi estático como el debido a la vibración; téngase presente que los valores cuasi estáticos son nulos para el caso de MU, pero no lo son para el caso de VE. En la figura se observa que la fuerza axial mínima para ambos arranques disminuyó para el caso VE, mientras que la compresión máxima aumentó, es decir, la VE incrementó el intervalo entre las fuerzas axiales mínima y máxima debidas a la 
combinación de carga permanente más sismo; la compresión mínima en el arranque izquierdo disminuyó $47 \%$, mientras que la máxima aumentó $15 \%$ para el arranque derecho. En la misma figura se presentan también las rotaciones en los arranques debidas al movimiento fuera del plano del puente y al movimiento en el plano. Fuera del plano la rotación del arranque izquierdo se redujo19\%, mientras que la del derecho aumentó 35\%. Las rotaciones en ambos arranques aumentaron notablemente para movimientos en el plano: la del izquierdo se incrementó 124\%, mientras que la del derecho lo hizo en $66 \%$; este hecho es atribuible al sustancial incremento de los desplazamientos verticales (figura 20) que afectan directamente a ambos arranques del arco. Los resultados obtenidos refuerzan la propuesta del Eurocódigo de evaluar el efecto de la variabilidad espacial en puentes con longitudes mayores que $600 \mathrm{~m}$.

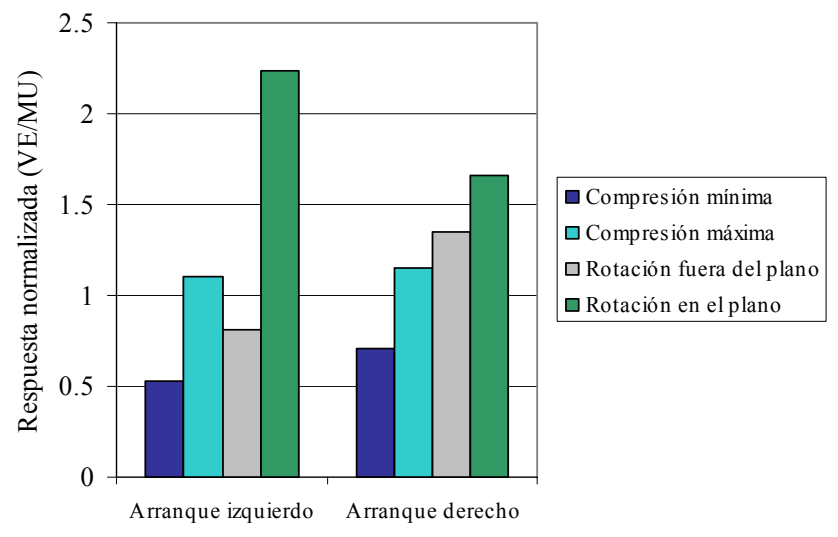

Figura 21. Respuesta de los arranques del arco considerando variabilidad espacial. Sismo de Campano-Lucano, estación Sturno

\section{CONCLUSIONES}

Como se ha podido observar, los arcos son estructuras que exhiben un comportamiento complejo durante la ocurrencia de movimientos sísmicos fuertes $\mathrm{y}$, para evaluar su respuesta de manera objetiva, debe recurrirse a técnicas de análisis dinámico no lineal. A la luz de los resultados obtenidos, mismos que se derivan de las hipótesis establecidas para el modelo analítico, son de destacar las siguientes conclusiones.

El periodo fundamental del puente resultó de $3.81 \mathrm{~s}$, que es muy superior al que suele tener un terreno duro, donde generalmente se desplantan este tipo de estructuras. Esto resulta particularmente favorable, ya que al no tenerse periodos similares entre la estructura y el terreno de apoyo, se está lejos de la posibilidad de una amplificación importante de la respuesta, además de que las aceleraciones espectrales de diseño son pequeñas para estructuras con periodos largos. 
Al evaluar la respuesta del modelo se obtuvo que los mayores desplazamientos de la estructura y, por ende las mayores demandas rotacionales en los elementos de la subestructura, fueron originadas por los sismos con mayores $v_{\text {máx }}$. Por ello, para predecir la respuesta de puentes con periodo largo es recomendable tener en cuenta a la $v_{\text {máx }}$ como medida de la peligrosidad.

Al evaluar las demandas medias de ductilidad rotacional generadas por los 10 eventos sísmicos se obtuvo que, para movimiento fuera del plano del modelo, solamente los arranques del arco vieron excedida su capacidad rotacional en $28 \%$ en promedio. Para movimiento en el plano del puente, la demanda media en los arranques del arco solamente alcanzó $24 \%$ de su capacidad rotacional, lo que es una consecuencia benéfica de la vinculación tablero/arco, que permite el deslizamiento longitudinal entre ambos elementos, eliminando la transferencia de fuerzas de inercia de la superestructura al arco. No obstante, los extremos de las pilas vieron rebasada por amplio margen su capacidad rotacional, hasta en $143 \%$ para el extremo inferior de la pila P4, poniendo de manifiesto la conveniencia de reducir la respuesta longitudinal del modelo. Las grandes demandas rotacionales en la subestructura de este tipo de puentes pueden abatirse empleando otra configuración estructural, o bien, mediante el uso de dispositivos de control pasivo.

La fuerza normal de compresión producida por la carga permanente en los arranques del arco tuvo una fluctuación media de $\pm 50 \%$ durante la ocurrencia de los movimientos sísmicos, por lo que resulta deseable considerar la interacción no lineal entre dicha fuerza y el momento flexionante. Esta interacción puede tenerse en cuenta de manera aproximada utilizando la metodología aquí presentada, o bien de manera más refinada modelando la estructura mediante elementos finitos tipo fibra.

Se exploró la influencia de la variabilidad espacial del movimiento sísmico del terreno considerando el efecto del paso de onda. Al considerar el movimiento asincrónico de los apoyos producido por uno de los eventos sísmicos, las rotaciones en los arranques del arco aumentaron notablemente, respecto al caso de movimiento uniforme, para flexión en el plano del puente, aunque sin exceder su capacidad rotacional; la rotación media del arranque izquierdo se incrementó en 124\%. Este hecho está asociado con la mayor respuesta vertical del modelo al tomar en cuenta la variabilidad espacial. Debe tenerse presente que estos resultados se obtuvieron considerando solamente las diferencias en los tiempos de llegada de las ondas, es decir, no provienen de un análisis de la variabilidad espacial, que comprende, además del paso de las ondas, la incoherencia, la atenuación y el efecto de sitio.

\section{RECONOCIMIENTOS}

Este trabajo forma parte del proyecto "Respuesta sísmica de un puente en arco con amortiguamiento viscoso suplementario" financiado por PROMEP-SEP, y se desarrolla en las instalaciones del edificio de posgrado de la Facultad de Ingeniería Civil de la Universidad Michoacana de San Nicolás de Hidalgo por parte del cuerpo académico Ingeniería y Ciencias de la Tierra. 


\section{REFERENCIAS}

Abeysinghe, R S, E Gavaise, M Rosignoli y T Tzaveas (2002), "Pushover analysis of inelastic seismic behavior of Greveniotikos bridge", Journal of Bridge Engineering, Vol. 7, No. 2, pp. 115-126.

Álvarez, J J (2004), "Respuesta sísmica de puentes arco empleando amortiguamiento viscoso suplementario", Tesis doctoral, Departamento de Ingeniería del Terreno, Cartográfica y Geofísica, Universidad Politécnica de Cataluña.

Álvarez, J J y Á C Aparicio (2003), “Comportamiento sísmico de puentes arco. Un panorama del estado del conocimiento", Revista de Ingeniería Sísmica, No. 68, pp. 21-53.

American Association of State Highway and Transportation Officials (AASHTO) (1994), LRFD Bridge design specifications, Washington, DC

Comisión Federal de Electricidad (CFE) (1993), Manual de diseño de obras civiles. Diseño por sismo. Acciones, Instituto de Investigaciones Eléctricas, México.

Comité Europeo de Normalización (CEN) (1998a), Eurocódigo 8: Disposiciones para el proyecto de estructuras sismorresistentes. Parte 1-1: Reglas generales. Acciones sísmicas y requisitos generales de las estructuras, ENV 1998-1-1, Edición de la Asociación Española de Normalización y Certificación.

Comité Europeo de Normalización (CEN) (1998b), Eurocódigo 8: Disposiciones para el proyecto de estructuras sismorresistentes. Parte 2: Puentes, ENV 1998-2, Edición de la Asociación Española de Normalización y Certificación.

Computers and Structures, Inc. (CSI) (1997), SAP2000 nonlinear version 7.10, Structural Analysis Programs.

Fujino, Y, S Hashimoto y M Abe (2005), "Damage analysis of Hanshin Expressway Viaducts during 1995 Kobe Earthquake. I: Residual inclination of reinforced concrete piers", Journal of Bridge Engineering, Vol. 10, No. 1, pp. 45-53.

Japan Road Association (JRA) (1996), Specifications for highway bridges. Part V: seismic design, Tokio, Japón.

Kawashima, K y A Mizoguti (2000), "Seismic response of a reinforced concrete arch bridge", 12th World Conference on Earthquake Engineering, Auckland, Nueva Zelanda, Paper No. 1824, CD-ROM.

Kurama, Y C y K T Farrow (2003), "Ground motion scaling methods for different site conditions and structure characteristics", Earthquake Engineering and Structural Dynamics, Vol. 32, No. 15 , pp. $2425-2450$. 
McCallen, D, C Noble y M Hoehler (1999), "The seismic response of concrete arch bridges: with focus on the Bixby Creek Bridge, Carmel, California", Report No. UCRL-ID-134419, Livermore, California, Lawrence Livermore National Laboratory.

Ministerio de Fomento (MF) (2000), EHE, Instrucción de Hormigón Estructural, Comisión Permanente del Hormigón, España, 476 pp.

Muller, J (2001), “On design and construction of long span concrete arch bridges", $3^{\text {rd }}$ International Arch Bridges Conference: ARCH '01, París, pp. 17-26.

Nazmy, A S (2003), "Seismic response of arch bridges", Proceedings of the Institution of Civil Engineers, Bridge Engineering 156, Issue BE2, pp. 91-97.

Sakai, J y K Kawashima (2002), "Seismic response of a reinforced concrete arch bridge taking account of variation of axial force", 12th European Conference on Earthquake Engineering, Londres, Paper No. 252, CD-ROM.

Simunic, Z, J Bleiziffer y G Janjus (2002), "Seismic analysis of the Krk Bridge", 12th European Conference on Earthquake Engineering, Londres, Paper No. 275, CD-ROM.

Spielmann, A (2001), “An arch of 600 meters", $3^{\text {rd }}$ International Arch Bridges Conference: ARCH '01, París, pp. 683-692.

Torkamani, M A M y H E Lee (2002), "Dynamic behavior of steel deck tension-tied arch bridges to seismic excitation", Journal of Bridge Engineering, Vol. 7, No. 1, pp. 57-67.

Tso, W K, T J Zhu y A C Heidebrecht (1992), "Engineering implication of ground motion A/V ratio", Soil Dynamics and Earthquake Engineering, Vol. 11, pp. 133-144.

Usami, T, Z Lu, H Ge y T Kono (2004), "Seismic performance evaluation of steel arch bridges against major earthquakes. Part 1: Dynamic analysis approach", Earthquake Engineering and Structural Dynamics, Vol. 33, No. 14, pp. 1337-1354.

Virola, J (2005), "World's longest bridge spans",

URL: [http://www.hut.fi/Units/Departments/R/Bridge/longspan.html] 